\section{Regaining its Destiny}

Physics research has been supported by two myths: the cold war and the development of civilian applications. The former has vanished; the latter became reality: physics research has succeeded in producing an impressive number of valuable technological applications. So it is generally felt in society that there is no longer a reason to finance scientific research in physics.

Recent attempts to infuse money into physics research, such as the large projects in thermonuclear fusion, superconducting supercolliders and space research, have met with complete failure. Those who control the money no longer fear nuclear war and enjoy a wealth of by-products of science in the form of modern conveniences. And many more of the last are in store for a long time to come.

There are, of course, problems such as disease, famine, over-population, crime, and the weaknesses of democratic systems; and perhaps governments would not be too reluctant to pay to solve these problems. Unfortunately, physics cannot promise much in these directions. Poor people have not gained much apparent benefit from science, and, consequently, do not believe in it any more. So we have lost credit here - credit that we never really deserved. The middle classes, meanwhile, have no perception of such things; and they do not count anyway since politics is carried out by smaller and smaller groups.

So what will physicists be doing ?

People in general will be more and more attracted by the outward appearance of physics: the fascination surrounding scientific discoveries, the thrilling adventures of the human mind in unravelling the mysteries of Nature, the anecdotal lives of great physicists, and a few other exciting, romantic things of this sort; the minimal knowledge needed to switch on and off the electronic devices that make everyday life so sweet; and fashionable diplomas and degrees

As soon as it lowers its requirements, physics will become more of a cultural and educational pursuit. The only thing we shall be left with is the outer appearance,

M. Apostol is with the Department of Theoretical Physics, Institute of Atomic Physics, P.O. Box MG35, Magurele -Bucharest, Romania. and we shall peddle it. Many physicists will move into teaching in schools and universities, which will proliferate with small sizes and low standards. Some will remain in small laboratories, that will become more technical as opposed to science-oriented and will provide expertise to businesses. A few will enter the communications, food, transport and entertainment industries as well as other disciplines such as engineering, chemistry, biology, medicine, climate and geophysics, economics, and social sciences. All are more or less at a descriptive level. Analytical and technical skills and experience in a scientifically well-developed discipline will be, hopefully, an asset for developing these areas. But none of this has anything to do with scientific research: it only says what physicists will be doing soon, once they are no longer physicists.

But most of us still indulge ourselves in the illusion that things are continuing, or will do so again quite soon. Since the taste of money is sweet, and vanity an enormous driving force, we lack critical thinking. In trying to keep the dead alive we do the best we know: many irrelevant publications; almost daily announcements of breathtaking new discoveries which are often proved false the next day; large conferences in the hope that large numbers matter; schools at all seasons to achieve the impression that something important is on the way; membership of small, exclusive circles which pretend to be the only ones doing "politically correct" science.

\section{Cum grano salis}

Physics, being a science, is only partially applicable to human beings so it should be taken with a grain of salt.

While public money proved to be addictive for us as physicists, many of today's technological achievements nevertheless originated in our work, and the cost to society of this work was extremely low. In other words, we were too cheap and too useful. It is therefore saddening to see how much we physicists reveal bad habits, lack of judgment, arrogance and frustration. The fact is that there is simply less money for scientific research while excitement and adventure in science can no longer compete with those the entertainment industry is delivering, and acting is not a palatable strategy.
Physicists also lack common sense. Like others, we agitate in a confusing manner, trying frantically to do something, which, in the best cases, is only a sort of mimicry. So our journals publish incorrect or trivial, papers, while systematically rejecting the few good ones. We simply have to admit that we are not good enough. We are also too numerous. The scientific community nowadays is an order of magnitude larger than during the last three centuries, and yet there has been no basic advance in knowledge within our science since quantum mechanics.

\section{Why did things go wrong?}

We do not know why the business went wrong for most except a small minority of fortunate people. But we have learnt a lesson, which might help us become wiser. For now that things have become clearer we can see the foolish game we played with government-funded research.

When, after the Second World War, physicists and society decided to strike a deal by having physics research financed directly, when the great "factories of science", i.e., the research institutes, were established, neither side knew what one was selling and the other buying. The only fact, which served as an impressive credential for physicists, was the atomic bomb. But the object of the deal remains a confusing dream, borne out of a mixture of the desire for power, humanitarian ideals, enthusiasm, fear, promises of comfort and welfare, etc. Why should the deal have gone well when nobody knew where it was going ? Nobody could have possibly dreamt up the astonishing output: nuclear reactors, microelectronics, the laser, magnetic resonance, magnetic memories, the electronic microscope, molecular epitaxy, and high-temperature superconductivity.

There were two subtle motivations for the deal which rarely receive attention. One concerns physicists. Scientific research is a continuous oscillatory motion between belief and disbelief, at the border between knowledge and ignorance. Scientists know only that something should be, but the particular form it may take they know not. Consequently, they will always be tempted to check their ideas to see if they are real. When society offered them the opportunity to do this, they rushed to strike a deal. They would do so again - and they would never be able to say exactly what they will come up with. The object of any bargain will always be largely unknown at the beginning. 
On the other hand, society invested a derisory amount of money in research, and this will always be the case whenever a similar opportunity arises. Scientific research is extremely cheap compared with other spending by society owing to the general lack of interest in research; there is not a large market for research, and they never will be.

The fact that so little was invested in science gave the second profound motivation for doing it. The risk was low. Most of the money has of course been lost. But even a large fraction of something small is still small. The remaining fraction, however, turned out to be extremely profitable. Economic competition should be understood as collecting the gains from scientific and technological achievements in the shortest possible time, for this was how a few fortunate people became richer and more powerful. Having invested in science, business became an extremely profitable venture. So profitable that one must now stop doing research and take the time to enjoy the rewards. The crisis that afflicts scientific research nowadays is a crisis of over-production.

\section{A lack of critical thinking}

As scientists we have lost much of our credit with the general public because the critical thinking which is proper to science is seldom, if ever, demonstrated when interacting with society. We share knowledge indiscriminately out of an urge to verify this knowledge, since openness is a very fruitful way of doing science. But this indiscrimination trades not only the knowledge we acquire today but also the entire stock inherited from past generations: when asking for funding for research we sell not only our own knowledge but the entire tradition of science. This throws a shadow upon our moral responsibility, or, at least, upon our judgment as regards the profitability of research. We never have bothered to provide guarantees for the future development of our science, even when we witnessed the high profitability of the output of our endeavours.

We also do not have the habit of stressing that science is still far from solving mankind's great problems. We do not spell out very often the basic tenets that define our profession.

Moreover, we lure beginners into scientific research without being able to tell them what is all about. Consequently, the collectivity of scientific researchers has increased indiscriminately over the years.
Meanwhile, society has discovered for itself another vocation, and there should be little surprise if the right to carry out scientific research becomes inscribed among human rights. People will increasingly want to come to science to see for themselves, through their own experience, what science is all about. One wonders of course if this will please them when they find out that science is the knowledge of our limits.

\section{What about future?}

In spite of the large amount of knowledge we have accumulated in many diverse domains, no new problem has emerged. On the contrary, while identifying new physical objects, we are continuously losing old, good problems that give our science its breadth. Announcements of new discoveries are announcements of false problems; there are many questions, but no new problems.

We have tested all the known ideas and concepts, and they work well. But we

\section{EPS Lecturer Foundations of Quantum Physics}

Anton Zeilinger, this year's EPS Lecturer, will address the foundations of quantum physics in a series of lectures presented at several institutes and universities throughout Europe. His programme includes a general lecture on the Experiments on the Foundations of Quantum Physics that is normally open to the general public. The more specialized talks are entitled:

- Multiparticle Entanglement and Nonlocality - Dynamical Diffraction of Atom and Neutron Waves in Periodic Media

- Principles of Quantum Communication and Quantum Computation.

Professor Zeilinger is presently the Director of the Institute for Experimental Physics, University of Innsbruck. He was educated in

\section{ERRATA}

The caption to the photograph accompanying the item "Lebanon: Encouraging Signs" [EN 27: 1 (1996) 28] referred to C. Sengör receiving the 1995 Rammal Medal from Lebanon's Minister of Culture and Higher Education. The caption should have read: "Miguel Virasoro, on the right, receiving the 1993 Rammal Medal from Michel Edde, Lebanon's Minister of Culture and Higher Education." In 1995 Miguel Virasoro succeeded Abdus Salam as Director of the International Centre for Theoretical Physics (ICTP) in Trieste". The Editor apologises for the error.

The item "A Mixed Profile" [EN 27: 2 (1995) 72] referred to E.W.A. Lingeman as the Physics and Society Action Committee's Secretary. He is in fact the committee's immediate past-chairman.

In the decisions of the 1996 Council [EN 27: 2 (1996) 77], the proposal that the Polish Physical Society take over the secretariat of the Mobility Scheme was made by the German Physical Society and not by the Polish society. are still left with the old puzzles (this does not mean that one should necessarily set out to solve them !): why force equals mass times acceleration, why entropy increases, why space is related to time, and why particles and waves are the same thing? We do not know; and who is asking ? Nobody asked Descartes, Galilei or Newton to explain motion; they were simply curious (and asking for money!). There was no apparent need to work out the chaotic motion of atoms; but Boltzmann did it, probably out of a sort of enthusiasm that may have been fatal. The practitioners of the electromagnetic equations were not at all aware of their missing the displacement current, with one exception: Maxwell. Without Bohr's stubbornness that something must work with electrons in atoms we would most likely not have had quantum mechanics.

Silently and with humility, and after the hubris that marked its interlude with society has faded away, physics will regain its destiny - that of Philosophiae Naturalis.

Austria and received a $\mathrm{PhD}$ from the Technical University, Vienna, before joining both the Massachusetts Institute of Technology, Cambridge, MA, USA, (1981-93) and the TU

Vienna, (1980-93) as an Associate Professor. He became Professor of Physics at the University of Innsbruck in 1993. Professor Zeilinger has held numerous visiting appointments in Europe, the USA and Australia, the most recent being as the Chairman and Scientific Director of the Nuclear Reactor Laboratory, TU Vienna, and Visiting Professor, Collège de France, Paris, and has authored some 150 scientific publications, mainly on the foundations of quantum physics.

For further information, please contact: Prof. A. Zeilinger, Institut für Experimentalphysik, Technikerstr. 25, A- 6020 Innsbruck. Tel.: +43-512-507 63 00; fax: +43-512-507 2921 ; anton.zeilinger@uibk.ac.at

1st European Conference on

Physics Teaching in Engineering Education PTEE 1997

4-6 June 1997

Engineering College of Copenhagen, Denmark

EPS Sponsored

Chair: E. Oehlenschlaeger (eoe@iktmail.cph.ih.dk)

Programme: university-level teaching strategies, media for physics teaching and physics curricula. Special topic: What do engineers and industry expect from physics teaching?

Registration: DM 200.-

Information:

L.O. Petersen

Ingenoemoejskolen, Koebenhavns Technikum

Lautrupvang 15, DK-2750 Ballerup

Tel. $+45(-) 44978088-\mathrm{Fax}+45(-) 44972700$

lop@cph.ih.dk 